generally arise when a low impedance path develops between the anode and cathode structures inside a pump, usually because a flake of titanium has broken off the anode and became lodged between it and the cathode, or because of the growth of a titanium whisker between the two electrodes.

The construction, function, characteristics, advantages, and disadvantages of ion pumps are discussed in some detail in my book "Vacuum Methods in Electron Microscopy". As noted there (p. 295), this problem can sometimes be alleviated, at least temporarily, by turning the power to the pump off carefully allowing the pressure in it to rise to just above $1 \mathrm{~Pa}(0.01 \mathrm{Torr})$, and then turning the power on again for a few seconds. This will generate a high intensity gas discharge inside the pump which may move the flake out of the offending position or destroy the whisker. Care must be taken not to allow the discharge to run for more than a few seconds, otherwise the pump or its power supply may be seriously damaged. It may be necessary to repeat this treatment three or four times to achieve the desired result, but if it is successful the pump's current will become stable again when the pump is put back into normal operation, and it may perform satisactorily for a considerable period of time. Ultimately, of course, such measures will no longer be effective and the pump will have to be rebuilt or replaced.

Wilbur C. Bigelow, University of Michigan

Professor Bigelow's book, "Vacuum Methods in Electron Microscopy", published by Portiand Press, London, is available in the U.S. from the Ashgate Publishing Company, Old Post Road, Brookfield, VT 05036, Tel: 800-535-9544; Fax: $802-2763837$. The price is $\$ 80$ in soft cover and $\$ 175$ in hard cover, plus a $\$ 3.75$ charge for shipping and handling.

\section{Making Formvar-Coated grids in High Humidity}

Make a trap door in the top of a (big) peanut butter jar, complete with hinges and rubber gasket, then put silica gel in the bottom of the jar, with a healthy layer of gauze. A tallish beaker is nestled into the silica gel and gauze, the beaker holding upright a Dip-Miser slide dipping beaker. Make up about 12 $\mathrm{mL}$ of Formvar, and put it into the Dip-Miser (the secret here is to buy a very concentrated solution and dilute it with ethylene dichloride, but that's just superstition at this point). (Note: use your solvent of choice, such as chloroform if preferred, ethylene chloride is not required for this.)

Sprinkle the gauze with ethylene dichloride to make an ethylenedichloride-vapor atmosphere which, as it evaporates, keeps the partial pressure of the solvent up and the humid air out. All of this can be done through the trap door.

Pre-dry the slides to be used over silica gel. Dip a clean, but not-tosqueaky-clean Corning non-frosted slide into the Formvar solution with long forceps and, holding the trap door partially shut, hold the slide in the ethylene dichloride atmosphere for 15 to 30 seconds while the Formvar begins to dry. It also thins during this step, so make a more concentrated solution than usual, about $1 \%$ or so.

Touch the end of the slide to the lip of the Dip-Mliser beaker to wick off the last drop, then quickly transfer the slide into a jar with silica gel and a round of filter paper. Prop the slide up against the side of the jar and quickly cover the jar and let the Formvar dry for several minutes.

Strip and float the coating onto water as usual, and lay the TEM grids on the floating films.

Pick up the floating films plus grids with Parafllm, wick them dry, and store them in covered Petri dishes. They release easily from the Parafllm.

Tina (Weatherby) Carvalho, University of Hawaii

If you start with Formvar powder or other solid form, the best practice is to $\mathrm{dry}$ the it at $60^{\circ} \mathrm{C}$ for half an hour just before mixing it into the solvent.

Fractionate the solvent first. Redistill it and monitor the temperature of the vapor at the stillhead and discard the (watery) fraction that comes off before the correct boiling point of the solvent being used is reached. Stop the still when there is around an (oily) tenth remaining of the original solvent.

Mel Dickson, University of New South Wales

\section{A Thin Film Casting Hint, Useful For Formar}

Thin films cast on glass slides out of dilute solutions in solvents are very useful in transmission microscopy, but you need to be able to get the film off. There are two approaches:

1. Get the slide REALLY CLEAN. Use really good gas burners and heat up a slide in a good hot flame until the edges start to round off and the slide would distort a bit. Then cool and dip the slide, and as soon as the solvent evaporates, scratch the edges and dip it into water vertically. The films on both sides strip right off on the water. BUT the slide has to be the right glass or it will crack up with heating/cooling stress and your flame has to be a generous size to avoid local overheating. Lovely if it works.

2. Get the slide REALLY DIRTY. The answer is at your fingertips. Or under your nose. Get a slide and put your unwashed thumb and finger on it. Got a nice greasy print on each side? No? Rub your thumb and finger on your nose. Now try. Good print, eh? Now polish the prints with a tissue so they can't be seen any more. Blow the tissue fibers off with a duster and dip the slide. Once the film is dry (about 5 minutes), puff some aspirated moisture over the slide. Strip the film right after it is dry. This usually works, but never leave the slide to dry too long or it won't strip.

Mel Dickson, University of New South Wales

\section{Glue for Sticking Sections to Grids (or each other)}

The traditional way to glue sections onto grids is to use scotch tape glue. Take about 2 feet of scotch tape from a roll fresh out of the packet to ensure the edges are free of dust. (Or cut off the edges from 2 feet of tacky self adhesive tape - take your pick.). Has to be cellulose clear (yellowish) tape which will not dissolve in solvent. Its not easy to find a tape these days in which the backing does not melt or dissolve in the solvent. For instance, Scotch 810 "Magic Transparent" is no good, in fact no scotch brand tape was suitable. But (in Australia) we can buy "Sellotape" brand which is labeled "Cellulose Tape". It is pale yellow transparent tape. Gib Ahlstrand reports that "Bemis" brand packing tape which is made in Minnesota works without dissolving the backing. (This means we have a case where cheaper is better - MT).

Put $100 \mathrm{ml}$ of chloroform in a brown bottle (reduces photolysis) and somehow poke the tape down the bottleneck. The tape should not change in appearance but the glue dissolves into the chloroform if you leave it for 48 hours. If the tape goes milky or shrivels up try a different kind. You don't want to use tape solution, just glue.

The grids as supplied are hydrophobic. I make them hydrophilic by whisking them through a small (spirit lamp) flame. They flash red and change color. If they shrivel, whisk faster. Place flamed grids on filter paper, put a drop of glue in chloroform on each grid, leave to dry, use right away. OR dip each grid in the glue bottle.

Don't sniff the glue!

\section{Mel Dickson, University of New South Wales}

This glue may also be used to help make serial-section ribbons. Wet a toothpick in the glue, swipe a tiny amount of the glue on the top and bottom faces of your "trimmed" pyramid, and allow to dry. Swipe from bottom to top, so that you can avoid getting any glue on the block face. Each section will then have a bit of the glue on its top and bottom edge as it comes off of the block face, and these edges will stick together, making a ribbon. - MT

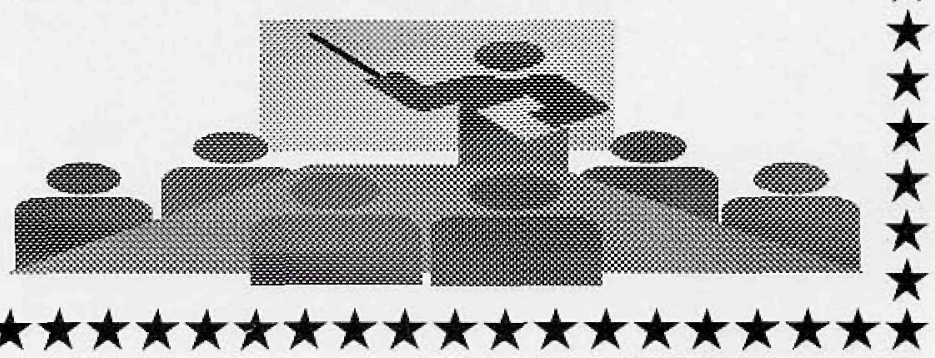

